\title{
The real world and thinking of thyroid cancer in China
}

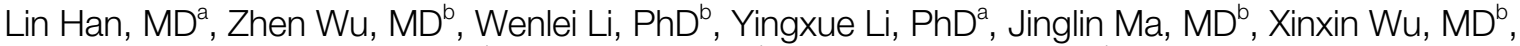 \\ Wenjuan Wen, MDª , Rui Li, MDº, Yumin Yao, MD ${ }^{b}$, Yongkun Wang, MD ${ }^{b, *}$
}

\begin{abstract}
Summary: The incidence of thyroid cancer has increased year by year, which has a major impact on the physical and mental health of patients. At the same time, it has a heavy psychological and economic burden on society and individuals. Based on the actual data of the thyroid cancer in Liaocheng People' Hospital in 2017, combine with the national and regional characteristics of China, this paper analyzes and discusses the controversy of initial thyroid operation modus.

Materials and methods: The clinical and pathologic data of 552 patients of thyroid cancer were collected from the department of thyroid surgery, who were initially discovered and treated surgically. 40 patients underwent endoscopic surgery, the range of resection was lobectomy + central lymph node dissection of the affected lobe. There were 512 cases underwent open operation with total thyroidectomy + central lymph node dissection, 239 of which were treated with neck lateral lymph node dissection at the same time.

Results: The overall metastasis rate of all patients was 59.42\%. Even the lymph node metastasis of papillary thyroid microcarcinoma was as high as $46.92 \%$. When the mass rose above $2 \mathrm{~cm}$, the proportion of metastasis increased to $77.53 \%$. When the tumor was complicated with bilateral and multiple high risk factor etc the proportion of metastasis were $63.54 \%$ and $71.19 \%$, respectively. Meanwhile, the incidence of contralateral accidental malignancy was $7.25 \%$ after postoperative paraffin pathology.

Conclusion: The malignant degree of thyroid cancer depends on the evolution of the tumor genome and there is a high rate of neck lymph node metastasis, especially when associated with high risk factors. It is recommended that at least total thyroidectomy + central lymph node dissection should be performed in initial treatment in China to avoid the risk of secondary operation and the burden of body and mind.
\end{abstract}

Keywords: Thyroid cancer, Metastasis, Surgery, Real world, Initial treatment

In the past 30 years, the incidence of thyroid cancer in the world has been increasing year by year, and the incidence of thyroid cancer in China has also been on the rise. In 2012, the number of new cases and deaths in China accounted for $15.6 \%$ and $13.8 \%$ of the world. By 2019, it is expected to become the third largest female cancer in the United States, with medical costs estimated at $\$ 190$ to $\$ 21$ billion, placing a heavy economic burden on society and individuals ${ }^{[1]}$.

In thyroid cancer, differentiated thyroid carcinoma accounts for $80 \%-90 \%$ of the cancer incidence. The most common metastasis is cervical lymph node metastasis, which seriously affects the

Departments of a Pathology and ${ }^{b}$ Thyroid Surgery, Liaocheng People's Hospital, Clinical Hospital of Taishan Medical University, Liaocheng, Shandong, China

This manuscript has been peer reviewed.

Sponsorships or competing interests that may be relevant to content are disclosed at the end of this article.

*Corresponding author. Address: Department of Thyroid Surgery, Liaocheng People's Hospital, Clinical Hospital of Taishan Medical University, Liaocheng 252000, Shandong, China. Tel/fax.: + 8606358272235 . E-mail address: wangyongkun923@163.com (Y. Wang).

Copyright (C) 2019 The Authors. Published by Wolters Kluwer Health, Inc. on behalf of IJS Publishing Group Ltd. This is an open access article distributed under the Creative Commons Attribution License 4.0 (CCBY), which permits unrestricted use, distribution, and reproduction in any medium, provided the original work is properly cited.

International Journal of Surgery Oncology (2019) 4:e81

Received 30 May 2019; Accepted 8 October 2019

Published online 6 December 2019

http://dx.doi.org/10.1097//J9.0000000000000081 prognosis of patients. Therefore, the effective and suitable surgical treatment for differentiated thyroid carcinoma is of great significance for clinical treatment.

Total and portion thyroidectomy are still the main treatment of primary surgery, so far, because there is no randomized large sample controlled clinical trial, so the comparison between the 2 mainly depends on retrospective analysis, there is controversy ${ }^{[2]}$. More than $50 \%$ of new papillary thyroid carcinoma (PTC) patients are thyroid micropapillary carcinoma with diameter $\leq 1$ $\mathrm{cm}$. In addition to micro-thyroid cancer, the incidence of thyroid cancer $>1 \mathrm{~cm}$ increased at the same rate. In addition, T3, T4 thyroid carcinoma with invasion of extraglandular tissue increased in the same trend $d^{[3]}$.

A total of 52,173 patients with PTC diameter $>1 \mathrm{~cm}$ in the National Cancer Database (NCDB), the mortality and recurrence rate of total thyroidectomy were lower in patients with lobectomy. A study of 22,724 patients with PTC using US surveillance, epidemiology and final results (SEER) database found no difference in survival rate between total thyroidectomy and lobectomy ${ }^{[4]}$. Domestic experts believe that papillary thyroid microcarcinoma (PTMC) is not a low risk, any advanced thyroid cancer is from the development of microcancer, any cancer that occurs at once is not a later stage of advanced PTC $^{[5]}$. The incidence of lymph node metastasis in the central region of PTMC was $20 \%-66 \%$. This indicates that although PTC progress is slow, but the ability and tendency to metastasize to lymph nodes and even distant organs are consistent with other malignancy. In generally, it is not scientific to classify malignancy according to tumor size. 
The ability of tumor to be invasive and distant metastases comes from the evolution of tumor genome ${ }^{[7]}$.

\section{Materials and methods}

A total of 552 cases of PTC treated and operated from January 1 to December 31, 2017 from Department of Thyroid Surgery, Liaocheng People's Hospital, Clinical Hospital of Taishan Medical University. All patients underwent fine-needle puncture before operation, with the pathology was papillary carcinoma or suspicious. A total of 105 cases were male and 447 cases were female, 13-72 years, there were 72 cases aged 35 years old and below.

Surgical procedures: 40 cases were operated by endoscopy, the range of lobectomy + homogenous central node dissection, the other 512 cases were treated with total thyroidectomy + central node dissection. Total thyroidectomy + central lymph node dissection + latral neck node dissection were performed in 239 cases.

The pathologic data were obtained from Department of Pathology, Liaocheng People's Hospital, Clinical Hospital of Taishan Medical University, were postoperative paraffin pathology.

\section{Results}

The incidence of metastasis was $59.42 \%$, and the proportion of metastasis was positively correlated with the diameter of tumor. The proportion of PTMC $\leq 1$ was $47.10 \%$, with the rate of lymph node metastasis was $46.92 \%$ (Table 1 ).

The proportion of bilateral cancer was 32.79 and the proportion of metastasis was 63.54 . The proportion of multiple cancer was 21.38 and the proportion of metastasis was 71.19. The contralateral lobe was negative before operation, and the malignant rate was $7.25 \%$ by accident. The proportion of lymph node metastasis was 32.5 and 39.84 , respectively, among lobectomy and total thyroidectomy. In all, $46.68 \%$ of the patients with total thyroidectomy underwent cervical lateral lymph node dissection, and the proportion of metastasis was $83.26 \%$. Only $7.95 \%$ of the patients had negative lymph nodes in the central region but lymph node metastasis in the lateral area of the neck. Only $16.74 \%$ of the patients had negative lymph nodes in the central region and the lateral area of the neck (Tables $2-4$ ).

In addition, we have counted the number of cases of thyroid cancer operation from our hospital in 2009-2018, the analysis results are on the rise year by year (Figs. $1-3$ ).

\section{Discussion}

According to the results of the SEER database of the National Cancer Center of the United States, not only did the incidence of

$$
\text { Table } 1
$$

The diameter and metastasis of thyroid cancer.

\begin{tabular}{lcccc}
\hline Diameter (cm) & Cases & Proportion (\%) & Metastasis & Proportion (\%) \\
\hline 0.5 & 94 & 17.03 & 42 & 44.68 \\
$\leq 1$ & 166 & 30.07 & 80 & 48.19 \\
$\leq 2$ & 191 & 34.60 & 118 & 61.78 \\
$>2$ & 89 & 16.12 & 69 & 77.53 \\
Total & & & 328 & 59.42 \\
\hline
\end{tabular}

Table 2

The surgery and high-risk factors of thyroid cancer.

Cases Proportion (\%) Metastasis Proportion (\%)

\begin{tabular}{lcccc}
\hline $\begin{array}{c}\text { Total thyroidectomy central } \\
\text { lymph node dissection }\end{array}$ & 512 & & 204 & 39.84 \\
$\begin{array}{l}\text { Lobectomy homogenous } \\
\quad \text { central node dissection }\end{array}$ & 40 & & 13 & 32.50 \\
$\begin{array}{l}\text { Bilateral cancer } \\
\text { Multiple cancer }\end{array}$ & 181 & 32.79 & 115 & 63.54 \\
Accidental cancer & 118 & 21.38 & 84 & 71.19 \\
\hline
\end{tabular}

PTMC below $1.0 \mathrm{~cm}$ increase from 1980 to 2010, but also the incidence of thyroid cancer of $1.0-4.0 \mathrm{~cm}$ also increased, especially among highly educated groups ${ }^{[8]}$. Statistics from the United States and South Korea show an increase in the incidence of thyroid cancer ${ }^{[9]}$. The results of surveys in Denmark, Finland, Israel, Japan, Spain, and Switzerland also showed an increase in the incidence of thyroid cancer, mainly PTC, and significant sex differences in the relative extent of the increase. This phenomenon cannot be fully explained by the increased sensitivity of testing methods or the increased awareness of doctors and patients in screening ${ }^{[10]}$.

Foreign scholars have actively carried out research on the risk factors related to thyroid cancer, found that autoimmunity, ionizing radiation, iodine intake, estrogen, environmental endocrine disruptors, negative psychosocial factors, and heredity may contribute to an increase in the incidence of thyroid cancer ${ }^{[11]}$.

From 1984 to 2010, the incidence of differentiated thyroid carcinoma in the United States increased. The diameter $\leq 0.5 \mathrm{~cm}$ thyroid carcinoma was $5.09 \%$, and the diameter of $0.5-0.99 \mathrm{~cm}$ thyroid carcinoma was $8.45 \%$, the average diameter of 1.0-1.99 $\mathrm{cm}$ thyroid carcinoma was $3.42 \%$, the diameter $\geq 2.0 \mathrm{~cm}$ of thyroid carcinoma was $2.96 \%$. This suggests that most PTMC will develop above diameter $1 \mathrm{~cm}$, but for varying periods of time. In particular, the possibility of dedifferentiation increases with the prolongation of the tumor-bearing time and the age of the patient. We think that with the extension of time, the malignant degree and the disdifferentiation of PTC are generally recognized, but when and what causes the malignant degree variation of the tumor genome has not been fully and reasonably explained.

Because the therapeutic effect is good, there is no need for early diagnosis and surgical treatment, which is logically wrong and violates the basic principles of early detection, early diagnosis and early treatment of malignant tumors. In addition, following up and waiting for the diagnosis of malignancies to change before taking action, the patient-undefineds psychological pressure is

\section{Table 3}

Latral neck node dissection or not and metastasis of thyroid cancer.

\begin{tabular}{lcccc}
\hline & Cases & Proportion (\%) & Metastasis & Proportion (\%) \\
\hline $\begin{array}{c}\text { Total thyroidectomy central } \\
\text { lymph node dissection }\end{array}$ & 279 & 54.49 & 119 & 42.65 \\
$\begin{array}{c}\text { Total thyroidectomy central } \\
\text { lymph node dissection }\end{array}$ & 239 & 46.68 & 199 & 83.26 \\
$\quad$ latral neck node \\
dissection
\end{tabular}


Table 4

The central lymph node of thyroid cancer.

\begin{tabular}{lccc}
\hline Central Lymph Node & Latral Neck Lymph Node & Metastasis & Proportion (\%) \\
\hline Positive & Negative & 60 & 25.10 \\
Positive & Positive & 113 & 47.28 \\
Negative & Negative & 40 & 16.74 \\
Negative & Positive & 19 & 7.95 \\
\hline
\end{tabular}

undoubtedly enormous. Cancer is not "with Chinese characteristics." In 2014, the Sloan Catelyn Cancer Center in the United States launched a "wait-and-see" program for thyroid cancer ${ }^{[12]}$. Patients diagnosed with microcarcinoma of the thyroid can choose not to have it removed for a while, but to check it out regularly. However, the vast majority of PTMC patients do not hesitate to choose surgical treatment. Some of the patients who chose to observe ended up asking for surgery after a while, and doctors feared being sued for missing the best treatment time. Early treatment not only has good effect, but also has low risk and less complications. Once lymphatic metastases and distant lung, bone metastases occur, the cost of medical care often increases more than several times, even endangering the life.

According to our data, as the diameter of the mass increases, the proportion of lymph node metastasis increases. The proportion of micropapillary carcinoma $\leq 1$ is 47.10 , and the rate of lymph node metastasis is 46.92, which does not mean that the risk of microcarcinoma is low. Lymph node metastasis is also an indicator of malignancy. With the passage of time, the tumor size, the proportion and the number of lymph node metastasis will further increase, leading to the disease progression, the difficulty and risk of surgery, and the risk of recurrence and metastasis gradually increased. When the mass rises above $2 \mathrm{~cm}$, the lymph node metastasis ratio rises to 77.53 , indicating that thyroid papillary carcinoma is progressing, the malignant degree and the disdifferentiation increases, although there is no way to evaluate and predict it.

The proportion of bilateral cancer was 32.79 and the proportion of metastasis was 63.54. The proportion of multiple cancer was 21.38 and the proportion of metastasis was 71.19. When associated with high risk factors, such as bilateral lesions, multiple lesions, lymph node metastasis ratio will be significantly increased, suggesting that we more fully and comprehensively carry out preoperative clinical evaluation, in order to facilitate us to obtain more comprehensive patient disease information, in order to give patients a more reasonable way of operation. Color Doppler ultrasonography and CT were negative in the contralateral lobe before operation, and the proportion of malignant lesions was 7.25 by accident after operation. All of them were small lesions with $<0.1 \mathrm{~cm}$. 40 patients were found the

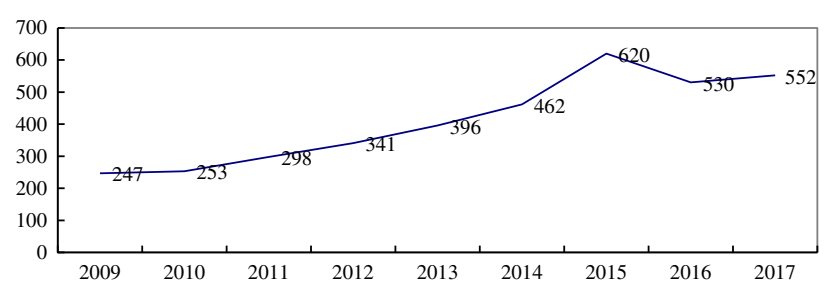

Figure 1. The number of cases of thyroid cancer in the past 9 years.

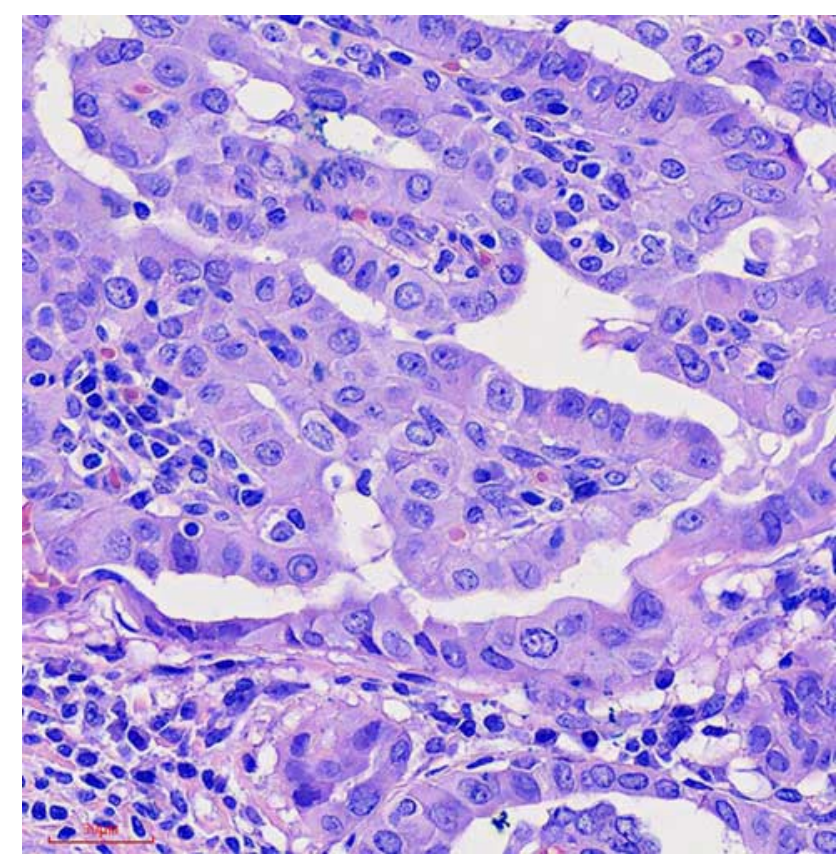

Figure 2. The classic type of PTC $\times 40$. PTC indicates papillary thyroid carcinoma.

contralateral malignant lesions accidentally after operation in this year. The results suggest that we still have defects in our full clinical impact assessment and may still omit malignant lesions.

Thyroidectomy can lead to complications, mainly parathyroid injury $(3 \%-5 \%)$ and recurrent laryngeal nerve injury $(1 \%-5 \%)$. It is acceptable that patients undergoing total thyroidectomy or partial thyroidectomy must use thyroxine replacement or even suppressive therapy for the rest of their lives ${ }^{[13]}$.

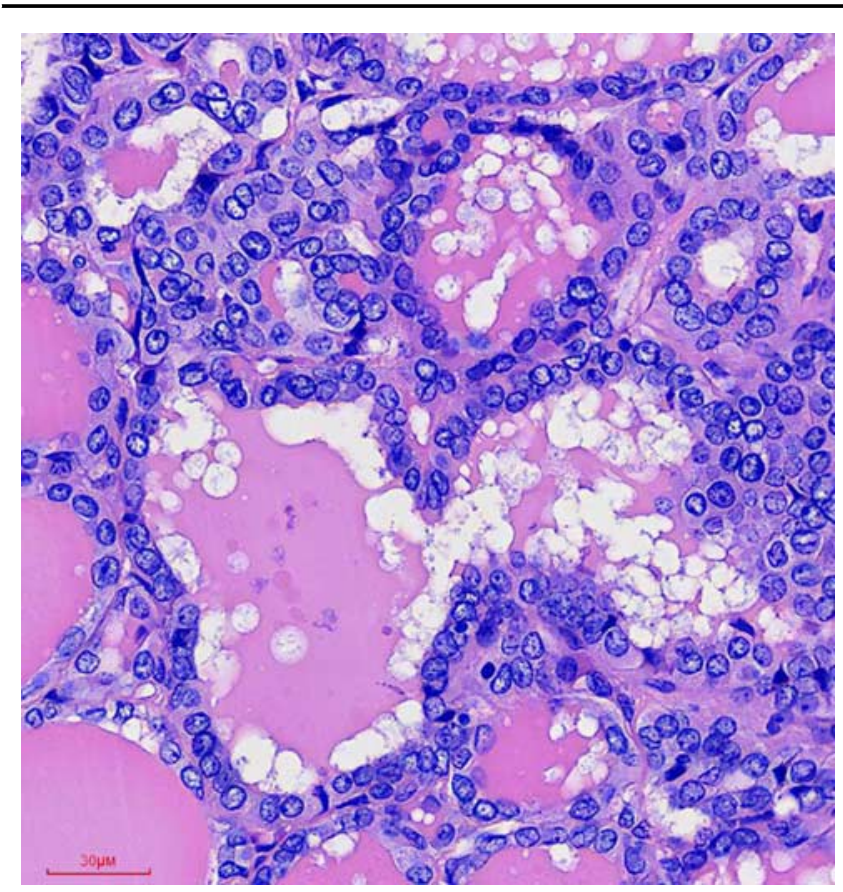

Figure 3 . The follicular variant of PTC $\times 40$. PTC indicates papillary thyroid carcinoma. 
We all find that the way thyroid cancer is treated is influenced not only by surgeons, but also by nuclear medicine, imaging intervention doctors, and even by health insurance companies. Careful preoperative stages and risk stratification of thyroid carcinoma will produce different results for thyroid carcinoma. This is more accurate surgery, more individualized and national trend. The author believes that the surgeon should firmly grasp the principle of tumor prevention and treatment, and remember that thyroid cancer is still a malignant tumor, and the scope of treatment should not be reduced at will, so as not to turn an operation into another operation several years later. Therefore, it is necessary to make an assessment according to the existing scientific level, according to the comprehensive judgment of the safety of the operation, the willingness of the patients and the medical resources, combined with the national conditions and regional factors of our country, and the degree of acceptance of the operation and the possible secondary operation by the patients. Make reasonable diagnosis and treatment plan. The data from our department remind us: (1) with the cases from our hospital during 2009-2018, the results are on the rise year by year (247-600). Thyroid cancer has become a common malignancy that threatens the patient's physical and mental health, witch will be heavy psychological shadow and economic burden on the individual and society. (2) Thyroid papillary carcinoma, even small papillary carcinoma, has a high rate of local metastasis $(59.42 \%$ and $46.92 \%)$, although it is an inert tumor after recovery, and its high invasiveness comes from the evolution of the evolution of tumor genome. Nor is it the low degree of malignancy that we do. (3) With the size of the tumor increasing, the local lymph node metastasis rate gradually increased, indicating that the invasiveness of the tumor became stronger. When the diameter of the tumor was larger than $2 \mathrm{~cm}$, the cervical lymph node metastasis rate increased to $77.53 \%$. It suggests that we should give more active and thorough treatment. (4) When the mass was complicated with bilateral lesions (63.54\%), multiple lesions $(71.19 \%)$ and other high risk factors, the tumor was highly invasive, suggesting that we should give priority to total central thyroidectomy lymph node dissection. Postoperative complications and long-term follow-up data will be followed up.

\section{Ethical approval}

All medical records are legal and reasonable. The study was approved and supervised by the ethics committee of Liaocheng People's Hospital, affiliated to Taishan Medical University.

\section{Sources of funding}

At present, there is no fund or subject support for this study.

\section{Authors' contribution}

All authors contributed equally, there is no sequence. Y.W. and L.H. are responsible for the study design. Z.W. and W.L. are responsible for data interpretation. Y.L. and L.H. are responsible for the manuscript writing. J.M. and X.W. are responsible for data collection. L.C. is responsible for literature search. R.L. and
Y.Y. are responsible for data analysis. All authors read and approved the final manuscript.

\section{Conflict of interest disclosures}

The authors declare that they have no financial conflict of interest with regard to the content of this report.

\section{Research registration unique identifying number (UIN)}

Researchregistry5257.

\section{Guarantor}

None.

\section{Acknowledgments}

The authors thank the Taishan Medical University and Liaocheng People's Hospital for supporting this study, and also thank to pathology and thyroid surgery for their support and assistance in this study. The authors thank for the hard work of everyone involved in this study.

\section{References}

[1] La Vecchia C, Malvezzi M, Bosetti C, et al. Thyroid cancer mortality and incidence: a global overview. Int J Cancer 2015;136:2187-95.

[2] Bilimoria K, Bentrem DJ, Ko CY, et al. Extent of surgery affects survival for papillary thyroid cancer. Ann Surg 2007;246:375-84.

[3] Vigneri R, Malandrino P, Vigneri P. The changing epidemiology of thyroid cancer: why is incidence increasing? Curr Opin Oncol 2015;27:1-7.

[4] Mendelsohn AH, Elashoff DA, Abemayor E, et al. Surgery for papillary thyroid carcinoma: is lobectomy enough? Arch Otolaryngol Head Neck Surg 2010;136:1055-61.

[5] Zhu J, Lei J. Overtreatment and deficiency of micropapillary thyroid carcinoma. Chin J Pract Surg 2016;36:520-3.

[6] Huang T. Standard diagnosis and treatment of micropapillary thyroid carcinoma: controversy, conclusion early. J Gen Clin Basis Chin 2016;23:12.

[7] Dong F, Zhang B, Shan G. Epidemic situation and influential factors of thyroid cancer in China. Chin Cancer J 2016;26:47-52.

[8] Li N, Du XL, Reitzel LR, et al. Impact of enhanced detection on the increase in thyroid cancer incidence in the United States: review of incidence trends by socioeconomic status within the surveillance, epidemiology, and end results registry, 1980-2008. Thyroid 2013;23:103-10.

[9] Ahn HS, Kim HJ, Welch HG. Korea's thyroid-cancer epidemic-screening and overdiagnosis. N Engl J Med 2014;371:1765-7.

[10] Pellegriti G, Frasca F, Regalbuto C, et al. Worldwide increasing incidence of thyroid cancer: update on epidemiology and risk factors. J Cancer Epidemiol 2013;2013:965212.

[11] Chen Y, Gao X, Cao M, et al. A case-control study of risk factors for thyroid cancer. Public Health in China 2014;30:955-7.

[12] Morris LG, Wong RJ, Tuttle RM. Ethical considerations when counseling patients with thyroid cancer about surgery vs observation. JAMA Otolaryngol Head Neck Surg 2016;142:406-7.

[13] Haugen BR, Alexander EK, Bible KC, et al. 2015 American Thyroid Association management guidelines for adult patients with thyroid nodules and differentiated thyroid cancer: the American Thyroid Association Guidelines Task Force on Thyroid Nodules and Differentiated Thyroid Cancer. Thyroid 2016;26:1-133. 